\title{
On the Roots of a Derivative of a Rational Function.
}

\author{
By L. R. Ford.
}

(Read 14th May 1915. Received 24th June 1915).

1. This paper extends some familiar theorems concerning the relations between the roots of a polynomial and those of its first derivative to the more general case of the rational function with a pole at a single point.

It is a well-known theorem that in the complex plane of the variable the smallest convex rectilinear polygon surrounding the roots of the polynomial surrounds also the roots of its first derivative." Proofs of this theorem have appeared recently in the Annals of Mathematics. $\dagger$ The extension of this theorem is made in the following section. The succeeding sections extend some further properties of polynomials, particularly those with real coefficients.

2. Let $f(z)$ be a function with a pole of the $n$th order at the finite point $a$, and with no other singularities. The most general form of the function is

$$
f(z)=\frac{b_{0} z^{m}+b_{1} z^{m-1}+\ldots+b_{m-1} z+b_{m}}{(z-a)^{n}}, \text { where } 0 \leq m \leq n, b_{0} \neq 0,
$$

and where the numerator does not contain $z-a$ as a factor. The function will have $n$ roots ; viz., the $m$ roots of the numerator, and $n-m$ roots at infinity.

Expressing $f(z)$ in partial fractions we have

$$
f(z)=\frac{A_{0}}{(z-a)^{n}}+\frac{A_{1}}{(z-a)^{n-1}}+\ldots+\frac{A_{n-1}}{z-a}+A_{n}, A_{0} \neq 0 .
$$

* Osgood: Lehrbuch der Funktionentheorie, Vol. 1, 2nd Ed., 1912, p. 211.

† Hayashi, in Vol. 15, p. 112, Maroh 1914.

Irwin, in Vol. 16, p. 138, March 1915. 
Now let the transformation $Z=\frac{1}{z-a}$ be made.

and

$$
f(z)=A_{0} Z^{n}+A_{1} Z^{n-1}+\ldots+A_{n}=F^{\prime}(Z)
$$

$$
f^{\prime}(z)=F^{\prime}(Z) \frac{d Z}{d z}=F^{\prime}(Z) \frac{-1}{(z-a)^{2}}
$$

If $Z_{i}$ is a root of $F(Z), z_{i}=a+\frac{1}{Z_{i}}$ is a root of $f(z)$; and if $Z_{i}^{\prime}$ is a root of $F^{\prime}(Z), z_{i}^{\prime}=a+\frac{1}{Z_{i}^{\prime}}$ is a root of $f(z)$. In addition $f^{\prime}(z)$ has two roots at infinity arising from the factor $\frac{1}{(z-a)^{2}}$. Laying aside these two roots at infinity, the roots of $f(z)$ and $f^{\prime}(z)$ are derived from those of $F(Z)$ and $F^{\prime \prime}(Z)$ respectively by the linear transformation $z=a+\frac{1}{Z}$.

$F(Z)$ is a polynomial, and to it we can apply the theorem mentioned above. The smallest convex rectilinear polygon in the $Z$-plane surrounding the roots of $F(Z)$ surrounds also the roots of $F^{\prime}(Z)$. Into what is this polygon transformed by the linear transformation $z=a+\frac{1}{Z} ? \quad$ It is a property of the linear transformation that a circle is transformed into a circle. When $Z=\infty, z=a$; hence the straight lines (circles through $\infty$ ) bounding the polygon become circles in the $z$-plane through the point $a$. To say that the polygon in the $Z$-plane is convex; i.e., has no reëntrant angle, is to make the requirement that the polygon is not divided into two parts by any of its sides, in other words, that the roots of $F(Z)$ lie all on the same side of each of the bounding lines of the polygon. The transformed region in the $z$-plane lies entirely within or entirely without each of its bounding circles.

The generalisation of the theorem is then as follows :-

Theorem. - If $f(z)$ is a rational function of $z$ whose only pole is at the point a, the smallest circular polygon surrounding the roots of $f(z)$-the sides of the polygon passing through a, and the polygon lying entirely without or entirely within each of its bounding circles - surrounds also the roots of $f^{\prime}(z)$, with the possible exception of two roots at infinity. 
Or this can be stated conveniently as follows:-Any circle through $a$, such that all the roots of $f(z)$ lie entirely on its exterior (interior), has also on its exterior (interior) the roots of $f^{\prime}(z)$, excepting the two at infinity. By constructing such circles through the roots we get the smallest possible polygon.

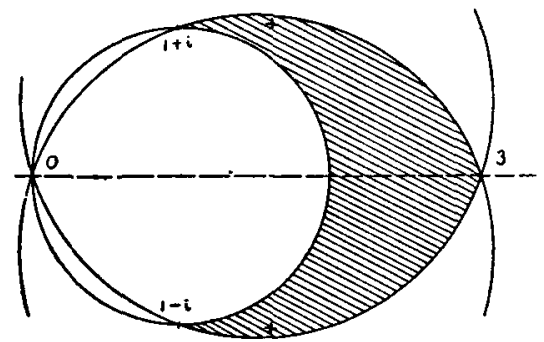

As an example, consider the function $f(z)=\frac{\left(z^{2}-2 z^{+}+2\right)(z-3)}{z^{3}}$, whose pole is $z=0$, and whose roots are $z=1+i, 1-i, 3$. The shaded region in the figure is the circular polygon of the theorem. The tinite roots of the derivative, $f^{\prime}(z)=\frac{5 z^{2}-16 z+18}{z^{4}}$, are marked by crosses.

The function $f(z)=c$ has a pole at the point $a$, and it has the same derivative as $f(z)$. Applying the theorem to this function we conclude that the roots of $f^{\prime}(z)$ lie within the polygon surronnding the $n$ points at which $f(z)$ takes on any given value.

3. The transformation $Z=\frac{1}{z-a}$ is only one of infinitely many linear transformations that can be used to make the transformed function a polynomial. It is essential only that when $z=a, Z=\infty$. We can, for example, make a transformation carrying $a$ to $\infty$ and any given circle through $a$ into the real axis. Such a transformation is

$$
Z=\frac{(c-a)(z-b)}{(c-b)(z-a)}
$$

where $b$ and $c$ are any two points on the given circle distinct from each other and from $a$. For the points $a, b, c$ are transformed respectively into $\infty, 0,1$; hence the circle through $a, b, c$ is 
transformed into the circle through $\infty, 0,1$; that is, into the real axis.

If all the roots of $f(z)$ lie on this circle through $a$, the roots of the transformed function, $F(Z)$, lie on the real axis. $F(Z)$ is then a polynomial whose coefficients are real, or at most contain a common complex factor; and the roots of $F^{\prime \prime}(Z)$ are real and alternate with those of $F(Z)$. As before, $\frac{d Z}{d z}$ has two roots at infinity. - Hence,

THEOREM. - If the roots of $f(z)$ lie on a circle through the pole, the roots of $f^{\prime}(z)$, excepting two at infinity, lie on the circle and alternate with those of $f(z)$.

It is a property of the linear transformation that points inverse with respect to a circle are transformed into points inverse with respect to the transformed circle. If there is a circle through a such that the roots of $f(z)$ either lie on the circle or are arranged in pairs inverse with respect to the circle, the roots of $F(Z)$, if the given circle be carried into the real axis, will be real or arranged in conjugate imaginary pairs. $F(Z)$ is a real polynomial; and its derivative has real or conjugate imaginary roots.

THeOREM.-If there exists a circle through the pole such that the the roots of $f(z)$ either lie on the circle or occur in pairs of points inverse with respect to the circle, then the roots of $f^{\prime}(z)$, excepting two at infinity, also either lie on the given circle or are arranged in pairs inverse with respect to it.

4. Of numerous other faets concerning polynomials which have analogues in the case of the function $f(z)$ one more will be mentioned. If the roots of $f(z)$ lie on a circle which does not pass through $a$, a transformation can be made carrying $a$ to infinity and the given circle into a circle with centre at the origin. If the roots of $F(Z)$ are equally spaced about this latter circle, the function is of the form $A Z^{n}+B$, and the roots of $F^{\prime \prime}(Z)$ are all at the origin. This yields the following :-

Theorem. - If the $n$ roots of $f(z)$ lie on a circle not passing through a, and if the circular arcs orthogonal to the given circle joining a to the roots divide the angle at a into $n$ equal parts, then $f^{\prime}(z)$ has, in addition to two roots at infinity, a single root of order $n-1$ at $a^{\prime}$, the point inverse to $a$ in the given circle. 